\title{
Missed Avulsion Fracture of the Ischial Tuberosity in an Adolescent Competitive Athlete: Case Report and Literature Review
}

\author{
Anastasia Pilichou ${ }^{1}$, Nick Sekouris ${ }^{1 *}$, Vasilios S Nikolaou ${ }^{2}$, Evangelos Demetriou ${ }^{3}$, Antonios Angoules ${ }^{3}$ and Stilianos Tsivgoulis ${ }^{4}$ and \\ Eugene Vlachos ${ }^{4}$
}

${ }^{1}$ Consultant Orthopeadic Surgeon, 'Metropolitan' General Hospital, Athens, Greece ${ }^{2}$ Medical School, Departmentt of Orthopedic Surgery, University of Athens, Athens, Greece

3'Metropolitan' General Hospital, Athens, Greece

${ }^{4}$ Department of Pediatric Orthopedics, 'KAT' Hospital, Athens, Greece

Keywords: Fracture; Trauma; Ischial tuberosity; Adolescent competitive athlete

\section{Introduction}

Hamstrings are among the most commonly injured muscles in athletes [1]. Injury at the hamstrings' origin, usually occurs when a sudden forcible contraction of the muscles against resistance is applied, causing excessive and eccentric overload. This type of trauma is common in various sporting activities.

Although the majority of these cases are muscle strains taking place most of the times in the musculotendinous junction [2], it is noticeable that a $12 \%$ of hamstring injuries may involve a tear or avulsion of the proximal hamstring origin and that another $9 \%$ represents complete avulsions [3]. Despite these well-documented cases of avulsions in the literature, there remains a diagnostic difficulty in differentiating a simple muscle strain from an avulsion. Therefore, patients continue to present late in the therapist for definitive treatment. Prompt diagnosis and subsequent treatment of ischial tuberosity avulsions are crucial, because the outcome in non-properly treated cases is a persistent and residual loss of muscle power.

\section{Case Presentation}

A 17-year-old soccer player referred to our clinic, due to persistent pain located at his right hip and at the posterior aspect of his thigh, 7 months after the initial injury. The trauma was elicited while he was performing a free kick, and he was immediately withdrawn from the match. The team physicians diagnosed initially hamstrings muscle strain and the patient was submitted in a course of physical therapy and rest for ten days. Afterwards, he was impelled to return to his previous sporting activities, but he was unable to follow the team's training program, as he continued to feel disabling pain every time he was flexing his hip with the knee in extension. The same physicians decided that he should undergo a new course of rest and physical therapy for a further period of two weeks. One and a half month after the initial injury, he returned to the training program, with his symptoms partly relieved. For the next four months, he was partially participating to the team's program with intervals of rest, as he still had pain in the back of his thigh, even in mild activities (e.g. running).

Due to the persistence of symptoms, his therapist decided an MRI scan of his thigh, in order to exclude any other pathology besides trauma. The MRI scans revealed areas of edema and hemorrhage, between obturator externus and ischial tuberosity, as well as around the sciatic nerve (Figure 1). The patient was then referred to our department, with the possible diagnosis of a neoplasm surrounding the sciatic nerve.

After a detailed history, he was submitted to a circumstantial clinical examination, there was detected prominent tenderness over the right ischial tuberosity, and a considerable decrease of his right hamstrings muscle's strength, comparing with the normal side. In the prone position, he could only partially extend the right hip. Apart from the decreased strength, there was also noticed a limitation in range of motion and flexibility. An antero-posterior pelvis radiograph was performed, which revealed a displaced fracture of the right ischial tuberosity (Figure 2). Since conservative treatment had failed, we informed the young patient and his parents that surgical intervention was necessary.

\section{Surgical Techniques}

Patient was placed in the prone position over chest rolls, and a transverse incision over the gluteal crease was made. The gluteus maximus muscle was mobilized and the sciatic nerve was identified and protected. The avulsed apophysis was exposed and cleaned of fibrous tissue (Figure 3). The fracture site was curetted, and the fracture fragments were reduced anatomically and fixated with three absorbable suture anchors. Before closing the surgical trauma, the integrity of the repair was evaluated by performing knee range of motion assessment. The wound was then irrigated and closed with absorbable sutures.

Directly, after the operation, patient was fitted with a harness,

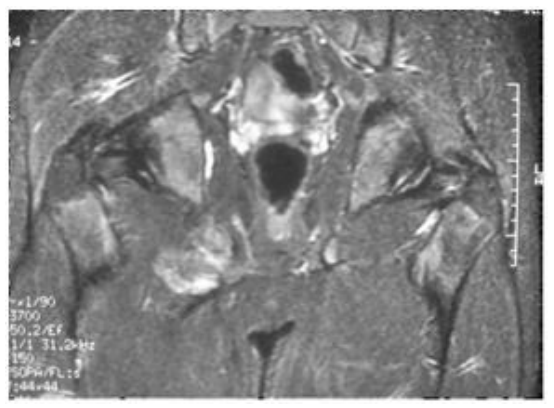

Figure 1: Anteroposterior (AP) X- ray of the pelvis, which demonstrates the dislocated fracture of the ischial tuberosity.

*Corresponding author: Nick Sekouris, Consultant Orthopeadic, 'Metropolitan' General Hospital, Athens, Greece, Tel: 0030 6985010730; E-mail: nick_sekouris@yahoo.com

Received March 02, 2014; Accepted March 27, 2014; Published March 29, 2014

Citation: Pilichou A, Sekouris N, Nikolaou VS, Demetriou E, Angoules A, et al. (2014) Missed Avulsion Fracture of the Ischial Tuberosity in an Adolescent Competitive Athlete: Case Report and Literature Review. J Trauma Treat S2: 002. doi:10.4172/2167-1222.S2-002

Copyright: (C) 2014 Pilichou A, et al. This is an open-access article distributed under the terms of the Creative Commons Attribution License, which permits unrestricted use, distribution, and reproduction in any medium, provided the original author and source are credited. 
Citation: Pilichou A, Sekouris N, Nikolaou VS, Demetriou E, Angoules A, et al. (2014) Missed Avulsion Fracture of the Ischial Tuberosity in an Adolescent Competitive Athlete: Case Report and Literature Review. J Trauma Treat S2: 002. doi:10.4172/2167-1222.S2-002

that kept the knee in flexion, and he was mobilized with crutches. The harness was applied for 4 weeks, and afterwards, he was allowed walking with partial weight bearing using crutches for another 4 weeks. Three months post-operatively there was evident to callus formation in the radiographs (Figure 4) and the patient started a physical therapy program, including a full range of motion exercises and muscle strengthening. At the end of the rehabilitation program, patient was completely relieved from his symptoms and five months after surgery, he was allowed to start jogging and gradually return to his previous sporting activities.

\section{Discussion}

Rupture of hamstring muscles is a common injury in athletes who in most cases is located at the muscle mass or at the myotendinous

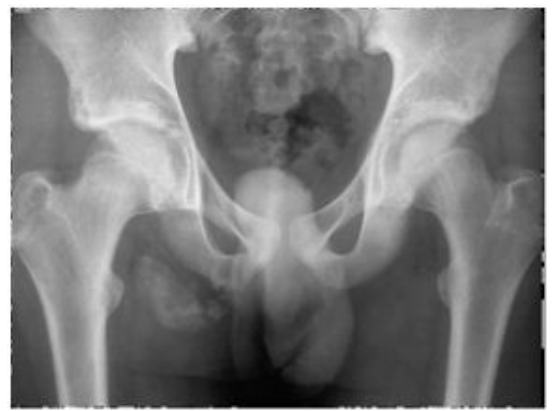

Figure 2: Patient underwent a Magnetic Resonance Imaging Scan (MRI scan) ten (10) weeks after the initial injury due to persistent pain during performance. In the region of the ischial tuberosity there are areas of edema and hemorrhage close to obturator externus, as well as around the sciatic nerve.

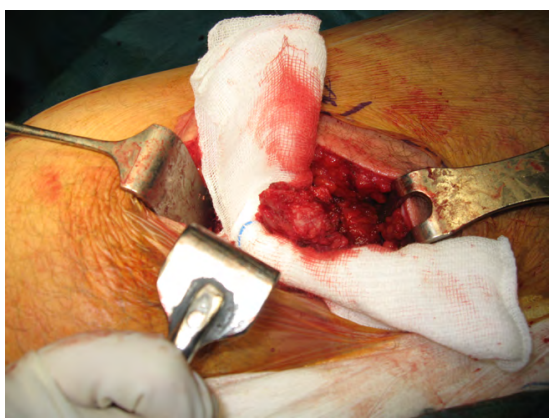

Figure 3: The avulsed apophysis was exposed and cleaned of fibrous tissue.

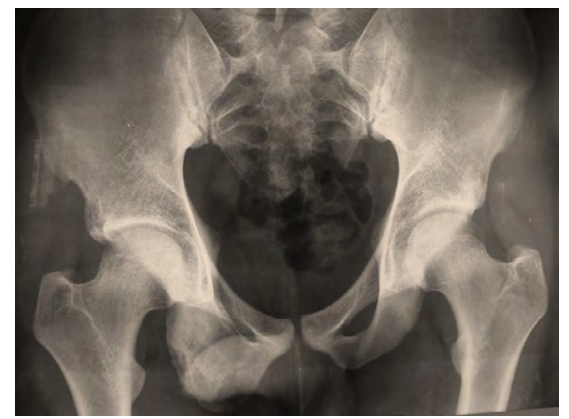

Figure 4: Three years postoperatively there was obvious radiologic evidence of callus formation. junction. The majority of hamstring injuries are strains of the muscle mass or at the myotendinous junction. Garret [4] has shown that in young athletes, hamstring muscle strains typically involve myotendinous disruption of the biceps femoris muscle, approximately $12 \mathrm{~cm}$ distal to the ischium. Complete rupture of the proximal origin of hamstring muscles is rare, comparing to mid-substance tears. Hamstring muscle tears if treated properly and non-operatively result in a satisfactory outcome [2]. Avulsion fracture of the hamstring origin from the ischial tuberosity occurs less commonly, and surgical intervention is necessary to accomplish a satisfactory functional outcome [5].

Ischial apophysis consists of a secondary ossification centre, which appears at puberty and does not fuse until the late teenage years or early twenties [6]. During this time period, when the tissue link between apophysis and bone is weaker than that between tendon and apophysis, a forcible contraction of the hamstring tendons may cause an avulsion of the apophysis itself. In general, the mechanism of injury is a forceful flexion of the hip with the knee extended while hamstring muscles are powerfully contracted [7]. Nevertheless, chronic repetitive traction across an open apophysis could also generate an avulsion. According to Rossi and Dragoni [8] the mean age of occurrence of ischium apophysis fractures is 13.8 years. Avulsion of the hamstring attachment from the ischial tuberosity in adults is rare. On the other hand, after the apophysis is closed in adults, the same mechanism of injury will yield a severe muscle tear rather than an avulsion [9].

Hamstring injuries are very common among athletes. In a study by Vandervliet et al. [10], the most frequent sports activities related to ischial tuberosity avulsion fractures seems to be gymnastics, soccer, fencing, tennis and running. In a series presented by Wood et al. [11] water skiing were found to be the most frequent cause of hamstring injury, whilst in their series Klingele et al. [5] reported sprinting as the most common mechanism of injury.

Historically, the first report of avulsion fracture of the ischial tuberosity was made by Berry [12] in 1912, concerning an English patient, who was treated surgically by removing the fragment. From then, until now, the condition has occasionally been reported in the literature. Ishikawa et al. [13] reported two cases of proximal hamstring tendon avulsion from the ischial tuberosity, without an associated fracture. Both patients underwent operative repair that resulted in full recovery and return to sports activities. Sallay et al. [14], reported 12 patients with severe avulsion injuries of the proximal hamstring tendon, who were treated conservatively. They concluded that these patients had a persistent and significant functional impairment. Koulouris G et al. [15] presented a series of 11 adult patients (range 21 to 51 years) with the diagnosis of complete proximal hamstring tendon rupture, whose treatment was early or late surgical. At the latest followup (average 34 months), they filled a questionnaire and were subjected in an isokinetic muscle test in order to estimate their functional ability. They concluded that there was no significant difference between early and late restoration and that an average of $91 \%$ recovery of hamstring muscle strength was achieved.

From all the cases cited in the literature, as well as from our clinical experience, it is excluded that the diagnosis of ischial tuberosity avulsion fractures is often delayed. The main reason is the consideration by the patient, the relatives and even the treating physician, that the trauma is trivial, while radiographs are not always performed. Early diagnosis requires a high index of suspicion as most of the times the history and the clinical presentation of avulsion of the ischial apophysis, closely mimics that of a simple hamstring strain. 
Citation: Pilichou A, Sekouris N, Nikolaou VS, Demetriou E, Angoules A, et al. (2014) Missed Avulsion Fracture of the Ischial Tuberosity in an Adolescent Competitive Athlete: Case Report and Literature Review. J Trauma Treat S2: 002. doi:10.4172/2167-1222.S2-002

Typically, patients describe the injury as the sensation of a sudden onset of pain in the posterior thigh or buttock with a subsequent gait disturbance. Patient may report feeling or hearing a "pop." Physical examination reveals posterior thigh pain distal to the ischial tuberosity and sometimes a palpable defect over that region, though this is difficult, as the injured part lies deep to the glutei muscles. Significant ecchymosis is present at the back of the thigh, because of the hematoma from the tendon rupture. Patients also report difficulty in sitting, walking, standing and squatting.

Imaging studies evaluation is fundamental for establishing an accurate diagnosis. Plain radiographs are usually negative, unless there is a bony avulsion from the ischial tuberosity. The osseous fragment seems to dislocate inferiorly and laterally to the ischial tuberosity. A Magnetic Resonance Imaging scan (MRI scan) is considered the gold standard for establishing the diagnosis of hamstring origin injuries [15]. It determines the amount of soft-tissue injuries, whether there is a complete or partial tear, the number of tendons ruptured and the amount of retraction. Some clinicians report Ultrasonography (US scan) as a useful diagnostic tool, but still its specificity and sensitivity for hamstring ruptures is not well established in the literature.

Treatment for proximal hamstring origin ruptures remains controversial depending on the level of athletic performance and the avulsion type. There are many reports in the literature of cases treated satisfactorily with rest, analgesics and cessation of activity until the symptoms subside [7]. Other authors suggest that it is mandatory, especially for athletically active patients to proceed with surgical treatment [7].

Salvi et al. [16] reported a case of spontaneous healing 5 years after initial trauma, with the persistence of pain while sitting and prolonged walking. In another study Bolgla et al. [17], present the case of an athlete with a proximal hamstring avulsion that was treated conservatively with a 5-phase gradually enforced rehabilitation program [9]. Four weeks after the injury, the athlete demonstrated normal hip range of motion and in eight weeks, the athlete's hip strength returned to a functional level. After successfully completing the therapeutic exercise, the athlete could return to his previous sporting level 14 weeks after the initial injury. Wootton et al. [18] recommended conservative treatment for avulsions with dislocation by less than $2 \mathrm{~cm}$. Early pain control and gait normalizing are the premium goals of rehabilitation. Once full range of motion and strength are restored, the athlete can begin training activities in preparation to full return to sports. A potential complication of conservative treatment is nonunion or the so-called "hamstring syndrome." This condition is characterized by shortening and usually fibrosis in the hamstrings leading to buttock pain, muscle weakness and decreased sporting ability. Surgical restoration of normal hamstring length is needed [18]. Complete tendon ruptures at the origin, especially in athletes require operative repair, because of the persistent weakness that is noticed in non-operated cases. It is preferred in general to treat the patient earl because of the poor results due to chronic fibrosis, retraction and atrophy reported in delayed treated cases [14].

There is a wide variety of surgical approaches and techniques described in the literature for treating the proximal origin hamstring avulsion fractures. According to some authors and for the cases of acute injury without any retraction, a transverse incision at the glutei crease with the patient in the prone position is preferred [19], which may provide an optimal cosmetic wound. Others preferred a longitudinal incision, which allows adequate identification and exposure of the hamstring tendons, which may be retracted to the thigh and sufficient exposure of the sciatic nerve [20] Sciatic nerve neurolysis should be considered whenever there is pain across its distribution or when there is noticeable reduce of muscle power [21].

The surgical technique applied by Wood [11] was through a longitudinal incision at the posterior thigh, with three anchors. According to the authors, substantially displaced, osseous avulsions of more than 1 to $2 \mathrm{~cm}$ should be reduced and fixed. The postoperative return to sports was noticed in $79 \%$ of the patients in six months.

In a series of 6 young patients [22], the surgical approach applied was a modified "Kocher-Langenbeck", which is a gold standard for posterior column acetabular fractures and only in a female patient the fracture was approached by a transverse incision in the glutei crease. The fixation consisted of pelvic reconstruction plates and screws augmented with bone graft. Postoperatively a non weight-bearing status was applied for 8 weeks and no splintage was needed according to the authors. The recent bibliography suggests that a cast of 90 -degree flexion should be used for 2 to 8 weeks following surgery to protect the repair [5]. The necessity of the splint could be an issue of future research.

Heinanen [23] describes a technique in which the hamstring tendons are approached through a transverse incision. After cleaning tendons and bone from scar tissue the tendons are reattached to the bone with three to five $2.8 \mathrm{~mm}$ anchors which are screwed to the bone in a shape of triangular, rectangular or X mode. The tendon is then pulled back to its anatomical position, and the sutures are tied. Afterwards a PDS-Cord suture is put through the sacrotuberal ligament just next to the bone and tightened and tied to keep the tension away from the suture anchors and to maximize the strength of attachment.

Marx et al. [24] report 2 cases of symptomatic chronic complete proximal hamstring tendon avulsions where inter-positional allograft tissue was used for reconstruction because the tendon was too retracted for primary repair. An Achilles tendon allograft was used in both cases. According to the authors, this procedure should be considered as a salvage operation as the patients did not return to a complete normal function and demonstrated hamstring weakness on the operated side.

Finally, Domb et al. [25] described a novel surgical technique for endoscopic repair of proximal hamstring avulsion injuries. Two working portals are used, direct posterior and postero-lateral, both in the gluteal fold. Additional portals may be added and used, primarily for anchor placement. According to the authors, endoscopic treatment offers a less invasive method to address proximal hamstring injuries and might decrease perioperative pain and complications. Advantages also include improved visualization of the anatomy, which may enable improved repairs as the technique is refined. Potential pitfalls include injury to neurovascular structures during blind portal placement, injury to the sciatic nerve if disoriented to arthroscopic anatomy, technical challenges of passing and shuttling suture for repair and increased operative time, particularly during early part of a learning curve. The authors believe that endoscopic repair can be beneficial, especially in acute repairs; however, further research on the role of endoscopic proximal hamstring repair is warranted.

\section{Conclusion}

Hamstring muscle injuries are common, especially in sports. They can be easily misdiagnosed resulting in impaired athletic performance and long rehabilitation times. A meticulous clinical examination and 
Citation: Pilichou A, Sekouris N, Nikolaou VS, Demetriou E, Angoules A, et al. (2014) Missed Avulsion Fracture of the Ischial Tuberosity in an Adolescent Competitive Athlete: Case Report and Literature Review. J Trauma Treat S2: 002. doi:10.4172/2167-1222.S2-002

a high index of suspicion are warranted, in order to avoid delayed diagnosis and proceed to a proper treatment protocol. The algorithm for the management of the individual should take into account that early operative treatment gives significantly better results than late surgery, and is therefore recommended. Despite this, considerable improvement of symptoms could also be achieved in chronic cases.

\section{References}

1. Bennell KL, Crossley K (1996) Musculoskeletal injuries in track and field: incidence, distribution and risk factors. Aust J Sci Med Sport 28: 69-75.

2. Järvinen TA, Järvinen TL, Kääriäinen $M$, Kalimo H, Järvinen M (2005) Muscle injuries: biology and treatment. Am J Sports Med 33: 745-764.

3. Koulouris G, Connell D (2003) Evaluation of the hamstring muscle complex following acute injury. Skeletal Radiol 32: 582-589.

4. Garrett WE Jr (1990) Muscle strain injuries: clinical and basic aspects. Med Sci Sports Exerc 22: 436-443.

5. Klingele KE, Sallay PI (2002) Surgical repair of complete proximal hamstring tendon rupture. Am J Sports Med 30: 742-747.

6. Bylak J1, Hutchinson MR (1998) Common sports injuries in young tennis players. Sports Med 26: 119-132.

7. Kujala UM, Orava S, Karpakka J, Leppävuori J, Mattila K (1997) Ischial tuberosity apophysitis and avulsion among athletes. Int J Sports Med 18: 149155

8. Rossi F, Dragoni S (2001) Acute avulsion fractures of the pelvis in adolescent competitive athletes: prevalence, location and sports distribution of 203 cases collected. Skeletal Radiol 30: 127-131.

9. Metzmaker JN, Pappas AM (1985) Avulsion fractures of the pelvis. Am J Sports Med 13: 349-358.

10. Vandervliet EJ, Vanhoenacker FM, Snoeckx A, Gielen JL, Van Dyck P, et al. (2007) Sports-related acute and chronic avulsion injuries in children and adolescents with special emphasis on tennis. Br J Sports Med 41: 827-831.

11. Wood DG, Packham I, Trikha SP, Linklater J (2008) Avulsion of the proximal hamstring origin. J Bone Joint Surg Am 90: 2365-2374.

12. Berry $\mathrm{J}$ (1912) Fracture of the tuberosity of the ischium. J Am Med Assn 1912:1450.
13. Ishikawa K, Kai K, Mizuta $\mathrm{H}$ (1988) Avulsion of the hamstring muscles from the ischial tuberosity. A report of two cases. ClinOrthopRelatRes : 153-155.

14. Sallay PI, Friedman RL, Coogan PG, Garrett WE (1996) Hamstring muscle injuries among water skiers. Functional outcome and prevention. Am J Sports Med 24: 130-136.

15. Koulouris G, Connell DA, Brukner P, Schneider-Kolsky M (2007) Magnetic resonance imaging parameters for assessing risk of recurrent hamstring injuries in elite athletes. Am J Sports Med 35: 1500-1506.

16. Salvi AE, Metelli GP, Corona M, Donini MT (2006) Spontaneous healing of an avulsed ischial tuberosity in a young football player. A case report. ActaOrthopBelg 72: 223-225.

17. Bolgla LA, Jones DL, Keskula DR, Duncan JB (2001) Hip Pain in a High School Football Player: A Case Report. J Athl Train 36: 81-84.

18. Wootton JR, Cross MJ, Holt KW (1990) Avulsion of the ischialapophysis. The case for open reduction and internal fixation. J Bone Joint Surg Br 72: 625-627.

19. Miller SL, Gill J, Webb GR (2007) The proximal origin of the hamstrings and surrounding anatomy encountered during repair. A cadaveric study. J Bone Joint Surg Am 89:44-48.

20. Lempainen L, Sarimo J, Orava S (2007) Recurrent and chronic complete ruptures of the proximal origin of the hamstring muscles repaired with fascia lataautograft augmentation. Arthroscopy 23: 441

21. Sallay PI, Ballard G, Hamersly S, Schrader M (2008) Subjective and functional outcomes following surgical repair of complete ruptures of the proximal hamstring complex. Orthopedics 31: 1092.

22. Gidwani S, Bircher MD (2007) Avulsion injuries of the hamstring origin - a series of 12 patients and management algorithm. Ann R CollSurgEngl 89: 394-399.

23. (2013 )Proximal Hamstring Avulsion - Anatomy, Cause of Injury, Surgical treatment and Post-operative Treatment Protocol Mikko Heinänen Suomen Ortopediaja Traumatologia Vol. 36 SOT .

24. Marx RG, Fives G, Chu SK, Daluiski A, Wolfe SW (2009) Allograft reconstruction for symptomatic chronic complete proximal hamstring tendon avulsion. Knee Surg Sports TraumatolArthrosc. 17: 19-23

25. Domb BG, Linder D, Sharp KG, Sadik A, Gerhardt MB (2013) Endoscopic repair of proximal hamstring avulsion. Arthrosc Tech 2: e35-39.

This article was originally published in a special issue, Trauma Injury and Orthopaedic Surgery handled by Editor(s). Dr. Robinson Esteves Santos Pires, Federal University of Minas Gerais, Brazil 